\title{
MEDULLARY THYROID CANCER A comparative study between sporadic and familial cases in a reference care center in Spain
}

\section{BACKGROUND}

Hereditary Medullary Thyroid Cancer (MTC) accounts for $20-30 \%$ of cases, and it is related to RET germline mutations and multiple endocrine neoplasia type 2 (MEN2). Our aim was to analyze differences in diagnosis and outcome between sporadic and familial cases of MTC in a cohort of patients.

\section{MATERIALS \& METHODS}

Retrospective, unicentric cohort study that included all genotyped patients with MTC $(n=48)$ diagnosed at Hospital Clínico San Carlos (Madrid) between 1984-2013; 42\% were germline mutation carriers (45\% moderate risk [category MOD], 45\% high risk [category $\mathrm{H}$ ], 10\% highest risk [category HST]). Median follow-up was 61 (IQR 22-104) months. A comparative analysis was performed using the Student's ttest, the chi-squared test and the log-rank test.

\section{RESULTS}

Mean age at diagnosis was 37.6 (SD 20.4) years in familial cases, and 62.5 (12.2) in sporadic cases $(p<.001)$; local or distant metastases were present in $44 \%$ of familial cases and $61 \%$ of sporadic cases $(p=N S)$. Most of the familial cases (55\%) were asymptomatic and diagnosed after genetic screening.

Six months after total thyroidectomy, $24 \%$ of sporadic cases and no familial cases showed progression. During follow-up, $27 \%$ of sporadic cases and $6 \%$ of familial cases developed distant metastases (median time 30 [856] months).

Progression-free survival (PFS) and distant metastasesfree survival (DMFS) were longer in familial cases $(p=N S)$. Attributable mortality due to MTC was $23 \%$ in sporadic cases and $0 \%$ in familial cases; overall survival (OS) was longer in familial cases $(p=.03)$.

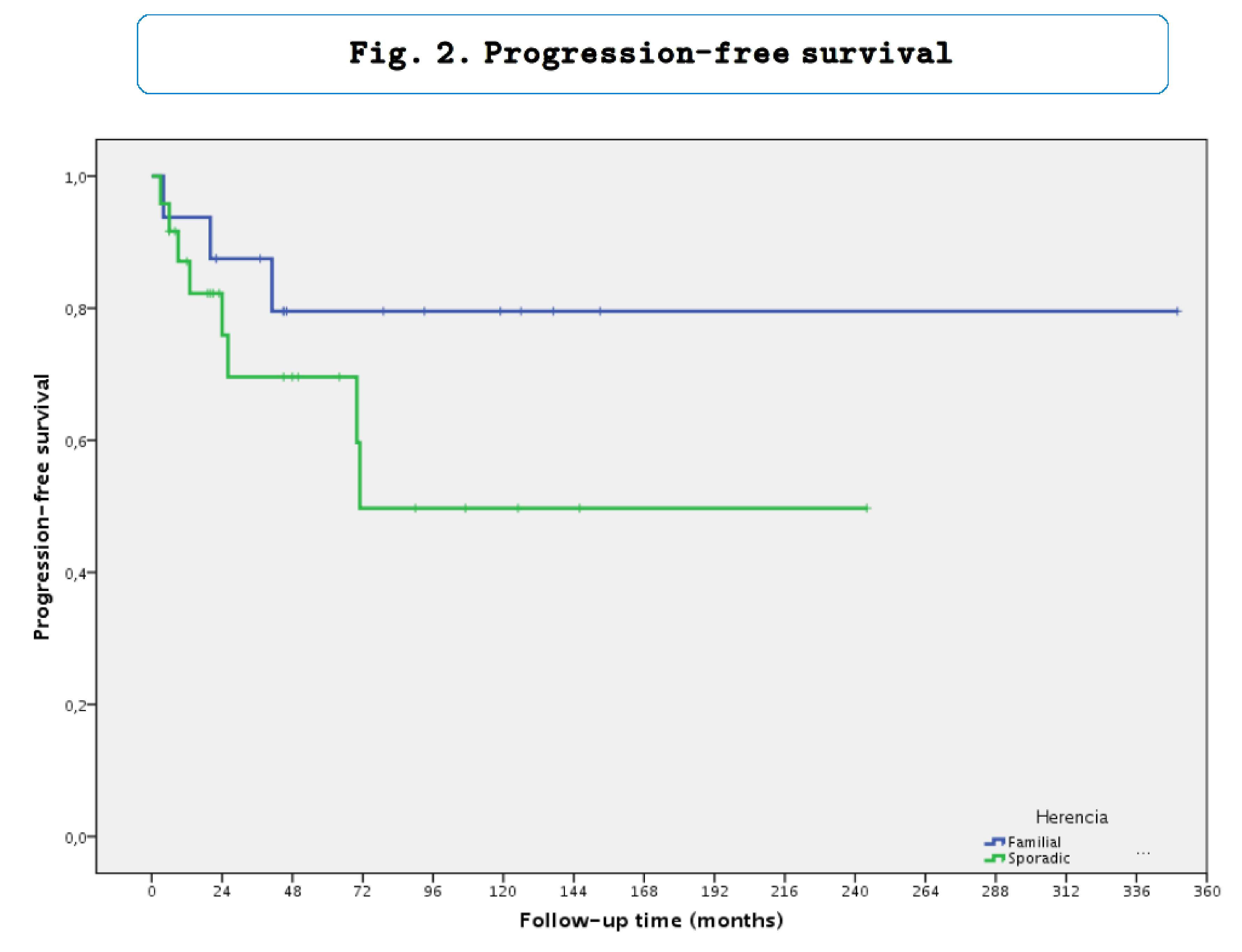

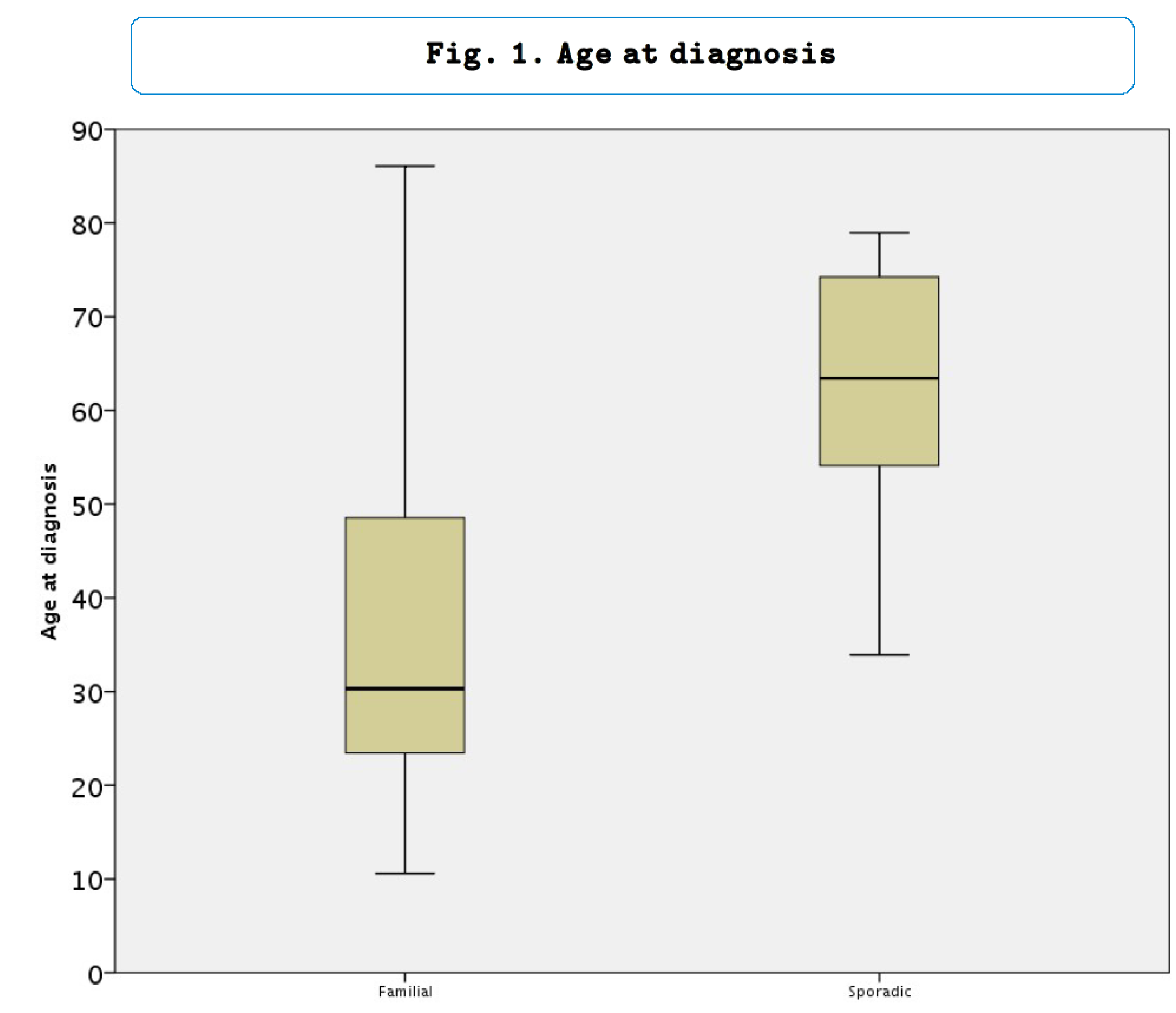

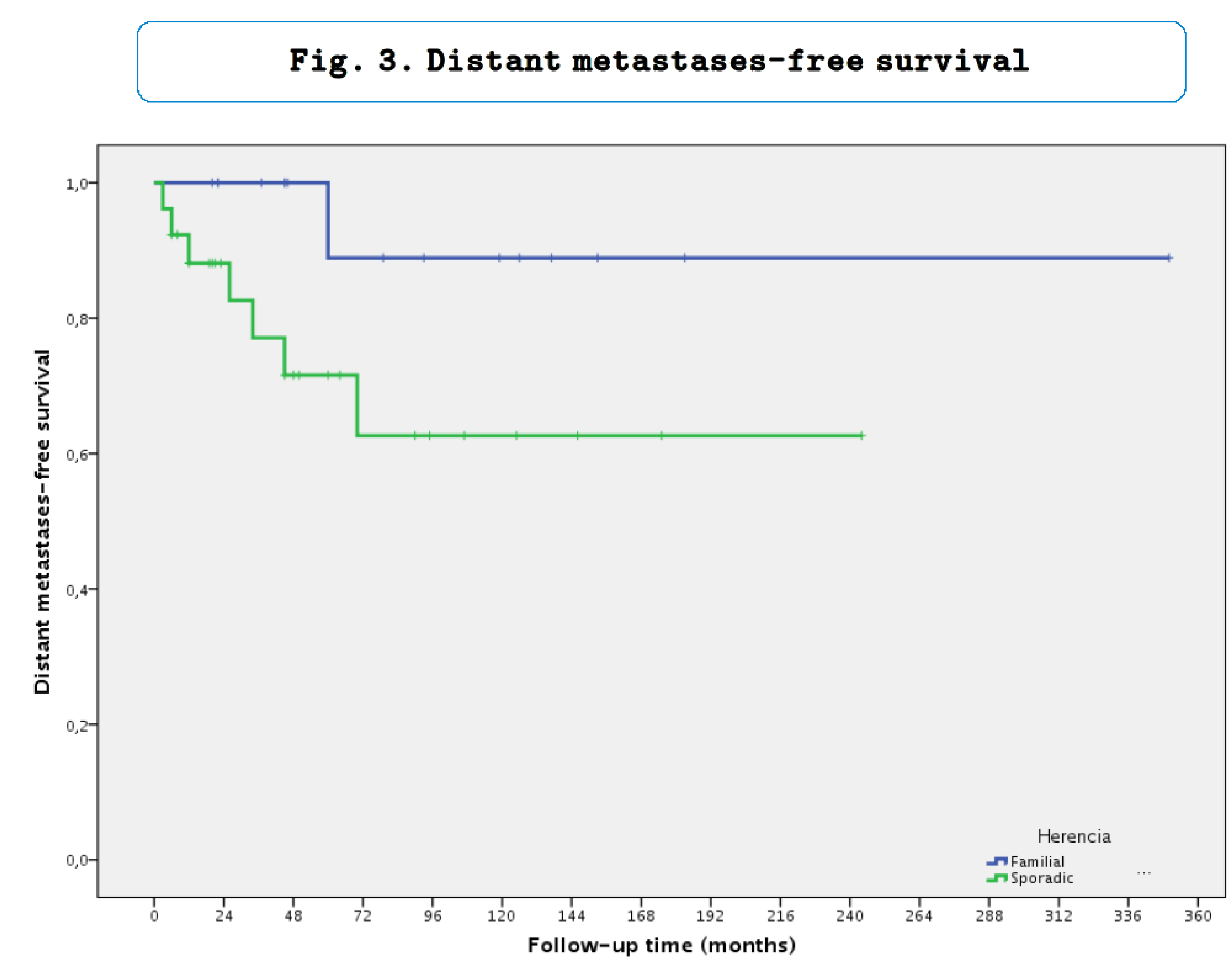

Age at diagnosis was significantly lower in familial cases of MTC. Genetic testing allowed for early diagnosis in asymptomatic mutation carriers, therefore familial cases had a better outcome and a longer survival. 